\title{
BREWING INDUSTRY RESEARCH FOUNDATION
}

\author{
NUTFIELD, SURREY
}

$\mathrm{T}$

HE new Brewing Industry Research Foundation at Lyttel Hall, Nutfield, Surrey, marks the fulfilment of a scheme developed over many years by the Institute of Brewing and made possible by the generosity of the Brewers' Society. It has been specifically designed for the scientific study of brewing and fermentation generally, with particular emphasis on the fundamental aspects of the many problems involved. Lyttel Hall itself has been skilfully modified and adapted to provide an extensive range of laboratories and other facilities in attractive surroundings. With its staff of scientific specialists and technicians under the direction of Sir Ian Heilbron, the Foundation occupies a unique place among industrial research institutes.

Not unexpectedly, versatile experimental breweries provide a focal point in the Foundation. Of these the larger, of ten gallons capacity and constructed of stainless steel, is complete with air-conditioned fermentation and beer-storage rooms, bottling plant, and pasteurizing equipment.

These breweries form part of an Applied Research Division designed to study, in the light of existing knowledge, the materials and processes of brewing as used to-day, but in greater depth and with greater accuracy than is possible in a commercial production plant. Further, this Division will assist the industry by formulating new or improved methods of analysis and also engage in general technological investigations covering all aspects of brewing.
Another important function of this Division is the much-needed examination of new varieties of barleys and hops for the National Institute of Agricultural Botany, Cambridge, and the hop research stations at Wye College and East Malling, Kent, as a preliminary measure of their brewing value. These tests include brewing trials of the more promising samples, and are of great value to the plant-breeder as they permit comparative tests to be made on small quantities of materials, thus saving valuable time and acreage.

The empiricism which for long characterized the traditional industries - and is, it must be borne in mind, still dominant in brewing - must inevitably be succeeded by scientific knowledge if full economy in the use of raw materials and complete control of operations is to be achieved. With this background, a wide range of studies is in progress in the other two main divisions of the Foundation. Of these, that devoted to microbiology forms a self-contained unit of three laboratories with a complete range of ancillary service rooms in which the most exacting studies of yeast and brewing bacteria can be carried out. The problems here are formidable, and of necessity begin with a survey of the almost unexplored field of British primary yeasts, a survey rendered peculiarly arduous by the difficulty of adequately characterizing and differentiating such yeasts. Similar difficulties complicate the complementary study of 'wild' yeasts - those adventitious

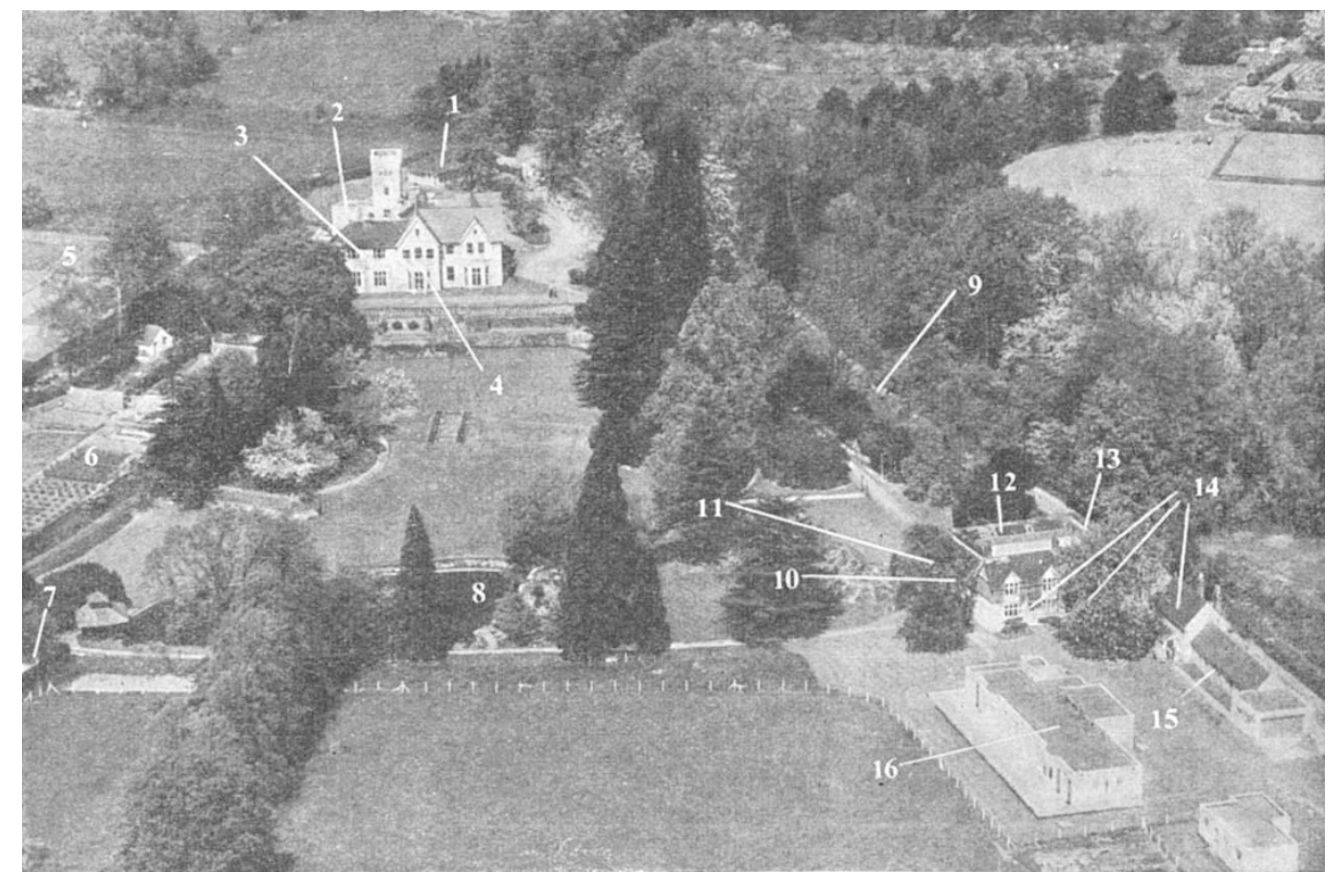

Brewing Industry Research Foundation, Lyttel Hall. 1, Lodge ; 2, model brewery ; 3, administration offices ; 4, main experimental block; 5 and 6 , kitchen gardens; 7, swimming pool ; 8, ornamental lake ; 9, Cooper's Hill Road ; 10 ,
electrophoresis laboratory ; 11, ultracentrifuge laboratory ; 12, light-scattering laboratory ; 13 , mechanics' workshop ; 14 , residential cottages ; 15 , ancillary workshops ; 16 , canteen 
contaminants which often have a deleterious effect on the beer. Again, beers are commonly susceptible to attack by certain types of bacteria, and although our knowledge here is more extensive than with the 'wild' yeasts, there is still need for further systematic study.

In this connexion the preservative action of hop resins assumes outstanding importance. Renewed attention is being given to means of estimating this effect by appropriate microbiological assay, a problem of peculiar difficulty because of the desirability that any test should reflect actual brewing conditions. The microbiological laboratories are also engaged on miscellaneous research such as the systematic examinations of the potential effects of selective herbicides and other agricultural chemicals on the malting quality of barley. Attention is also being directed to genetic studies on yeast, and to the possibilities of yeast hybridization. While many of these topics are of outstanding scientific interest, it must be emphasized that considerable research effort must perforce be devoted to essential but comparatively unspectacular groundwork. The need for this acutely reflects the sparse attention which has hitherto been paid to the scientific study of industrial microbiology, especially in Great Britain. For this reason an in stitute in which this largely neglected field receives prominence assumes special significance in view of the present meagre university facilities available for such studies.

Finally, mention should be made of the fact that in 1951 the Foundation undertook the important responsibility of maintaining the National Yeast Culture Collection. Some two hundred organisms were originally inherited from the National Collection of Type Cultures, and a further hundred and sixty yeasts of special interest have since been added. Work on the re-authentication of the cultures is already in progress, one-third of the total having been checked. Over the past year, nearly two hundred requests for yeasts have been received both from Britain and from abroad, and it is expected that this figure will increase as more yeasts are added to the Collection and its uses become more widely recognized. It will be appreciated that the National Yeast Culture Collection provides a service which extends far beyond the limits of the brewing industry.

The Chemical Division, which constitutes the third main section, comprises an extensive range of laboratories in which liberal facilities are provided for detailed research in the field of the industry's operations. Much thought has been given to the equipment of these laboratories, in which are installed all the apparatus, instruments and plant necessary to keep abreast of modern advances and techniques throughout the world. For example, the physical-chemical laboratories include the latest typo of air-driven ultracentrifuge, electrophoresis apparatus and light-scattering equipment, all indispensable for immediate and long-term research.

'The research programme covers the chemistry and biochemistry of the raw materials used in brewing and of the changes which they undergo during the various stages of the brewing process. Of these malt is pre-eminent, providing as it does the substance of beer, while hops supply the flavouring and preservative principles. An important early stage in malting consists of steeping the stored barley in water, an operation which it is now recognized results not merely in the uptake of water, but also in the simultaneous removal of a miscellany of substances. Among the latter are compounds which prevent or retard the normal germination of the grain. The isolation and study of these inhibitors are of considerable importance, for their planned use may conceivably lead to a higher degree of control of malting than has hitherto been possible; this aspect is now being investigated by means of specially designed experimental maltings. The barley grain is primarily a store-house for starches and proteins which are ultimately converted by specific enzymes into simpler compounds, such as dextrins and sugars on one hand and peptides and amino-acids on the other. A considerable effort is being devoted to the detailed study of these materials in barley, malt and wort, as well as to other constituents of the raw materials, notably pectins and tannins. Attention is a so being given to the major constituents of hops, more particularly the resins : although the importance of these has been appreciated for years, their full significance has still to be elucidated. This is evident as a result of the application of new techniques to their examination, whereby unsuspected constituents have been revealed.

In addition to these specific studies, many other brewing problems will ultimately only be interpreted as a result of fundamental scientific research. The formation of a 'head' on beer is thought to be associated with the presence of residual proteins; but their identification is still lacking and, moreover, there is complete ignorance of the physics and physical chemistry underlying the phenomenon. Again, the deposition of part of the residual proteins after processing frequently gives rise to the formation of an undesirable haze in the finished beer, and this is being given precise study in the hope ultimately of limiting the incidence of the responsible proteins.

In addition to its laboratory facilities, the Foundation houses the largest specialized brewing library in Great Britain. As part of its functions, it prints and distributes monthly to brewers throughout the country a bulletin of current literature which surveys, without abstracting, all incoming journals, thereby giving the brewing scientist notice of an article within four to six weeks of publication. The library also supplies on request photo-copies of scientific literature, a service which fills a long-felt want and is much appreciated.

This brief survey will serve to demonstrate that the Brewing Industry Research Foundation has not been set up solely to serve the immediate needs of an industry in respect of routine control and ad hoc problems, but equally for the prosecution of basic research, without which a full understanding of the vital processes involved can never be attained. The Director, appreciating the breadth of the field which must be covered and the many kinds of scientific experience required for such a task, has wisely taken steps to foster contacts with many university departments and, as occasion demands, to initiate therein extra-mural work.

The visitor to Lyttel Hall is left with a deep impression of an organization which, in its conception and in its whole scientific outlook, is destined not only to contribute substantially to industrial progress, but also, by its own contributions to fundamental knowledge, to take its place as a research centre worthy of the best traditions of Great Britain. 